\title{
WSI-Mindestlohnbericht 2010 - Unterschiedliche Strategien in der Krise
}

Vor dem Hintergrund der Weltwirtschaftkrise nehmen die gesetzlichen Mindestlöhne einen sehr unterschiedlichen Verlauf. Um die Unternehmen vor weiteren Kostensteigerungen zu bewahren, wurde in zahlreichen Ländern das bestehende Mindestlohnniveau eingefroren. Einige Länder haben hingegen moderate Erhöhungen vorgenommen, während wiederum in anderen Ländern der Mindestlohn stark angestiegen ist. Die unterschiedlichen Entwicklungen machen deutlich, dass es prinzipiell sehr verschiedene Wege gibt, um lohnpolitisch auf die Krise zu reagieren. Für eine Erhöhung der Mindestlöhne spricht, dass sie einen wichtigen Beitrag zur Stabilisierung der privaten Nachfrage und Abwehr deflationärer Tendenzen leisten.

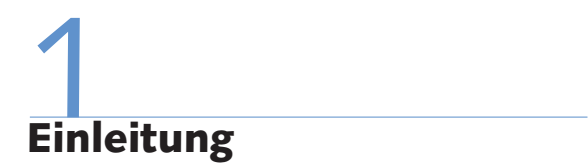

Gesetzliche Mindestlöhne gehören überall auf der Welt zu den etablierten Instrumenten zum Schutz der Beschäftigten und zur Regulierung des Arbeitsmarktes. In der Mindestlohndatenbank der Internationalen Arbeitsorganisation (ILO) werden über 100 Staaten aufgelistet, die eine oder mehrere gesetzliche Lohnuntergrenzen eingezogen haben, unterhalb derer keine legale Beschäftigung möglich ist (Eyraud/Saget 2005; ILO 2008). ${ }^{1}$ Hierzu gehören nicht nur die große Mehrheit der europäischen Länder, sondern auch alle führenden außereuropäischen Industriestaaten (wie z. B. Australien, Japan, Kanada und die USA), viele große aufstrebende Volkswirtschaften (wie z. B. Brasilien oder China) und zahlreiche Entwicklungsländer.

Im Hinblick auf ihr absolutes Niveau, ihre Kaufkraft, ihren relativen Wert im jeweils nationalen Lohngefüge sowie ihre Anpassungsmechanismen und Entwicklungsdynamiken weisen gesetzliche Mindestlöhne von Land zu Land jedoch große Unterschiede auf. Im Rahmen des WSIMindestlohnberichtes werden einmal jährlich die aktuellen Mindestlohnpolitiken

\section{Abb. 1: Gesetzliche Mindestlöhne pro Stunde 2010 - in Euro*-}

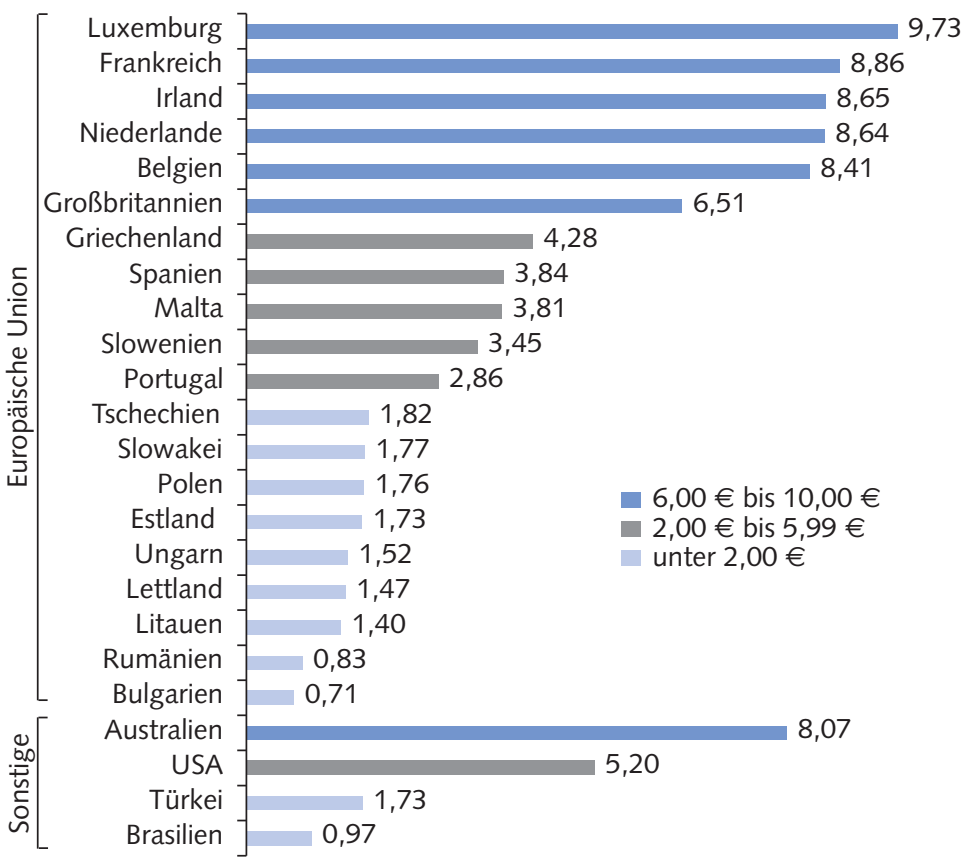

*jeweils zum 01.01.2010; Umrechnung in Euro zum Jahresdurchschnittskurs 2009. in ausgewählten Ländern analysiert und aktuelle Mindestlohndaten aufbereitet. Die Grundlage des Berichtes bildet die eigens hierfür aufgebaute WSI-Mindestlohndatenbank, die mittlerweile Daten für 24 Länder enthält. ${ }^{2}$ Im Mittelpunkt der Analyse stehen die Mitgliedstaaten der Europäischen Union (EU). Vor dem Hintergrund der globalen Wirtschaftskrise wurden darüber hinaus erstmals mit der Türkei ein EU-Beitrittskandidat und mit Australien, Brasilien und den USA drei außereuropäische Länder in die Untersuchung einbezogen.

\section{2 Gesetzliche Mindestlöhne zum 1. Januar 2010}

Bei den 20 von insgesamt 27 EU-Staaten, die über einen allgemeinen, branchenübergreifenden gesetzlichen Mindestlohn verfügen, lassen sich im Hinblick auf die absolute Höhe des Mindestlohns gemessen in Euro drei Gruppen identifizieren (Abbildung 1): Zur ersten Gruppe mit relativ ho-

\footnotetext{
1 Eine elektronische Version der ILO Mindestlohndatenbank mit aktuellen Updates findet sich unter: http://www.ilo.org/dyn/travail/travmain.home

2 Eine aktuelle elektronische Version der WSI-Mindestlohndatenbank findet sich unter: http://www. boeckler.de/pdf/ta mindestlohndatenbank.pdf. Zu den Unterschieden und Abgrenzungen der bestehenden Mindestlohndatenbanken von EUROSTAT, ILO, OECD und WSI vgI. Schulten (2009). Für die jüngste EUROSTAT-Auswertung über Mindestlöhne in Europa vgl. Czech (2009).
}

Thorsten Schulten, Dr. Wissenschaftler im WSI in der Hans-Böckler-Stiftung. Arbeitsschwerpunkte: Arbeits- und Tarifpolitik in Europa. e-mail: Thorsten-Schulten@boeckler.de 


\section{Abb. 2 Gesetzliche Mindestlöhne pro Stunde 2010}

- in Kauftraftstandards (KKS) ${ }^{*}$

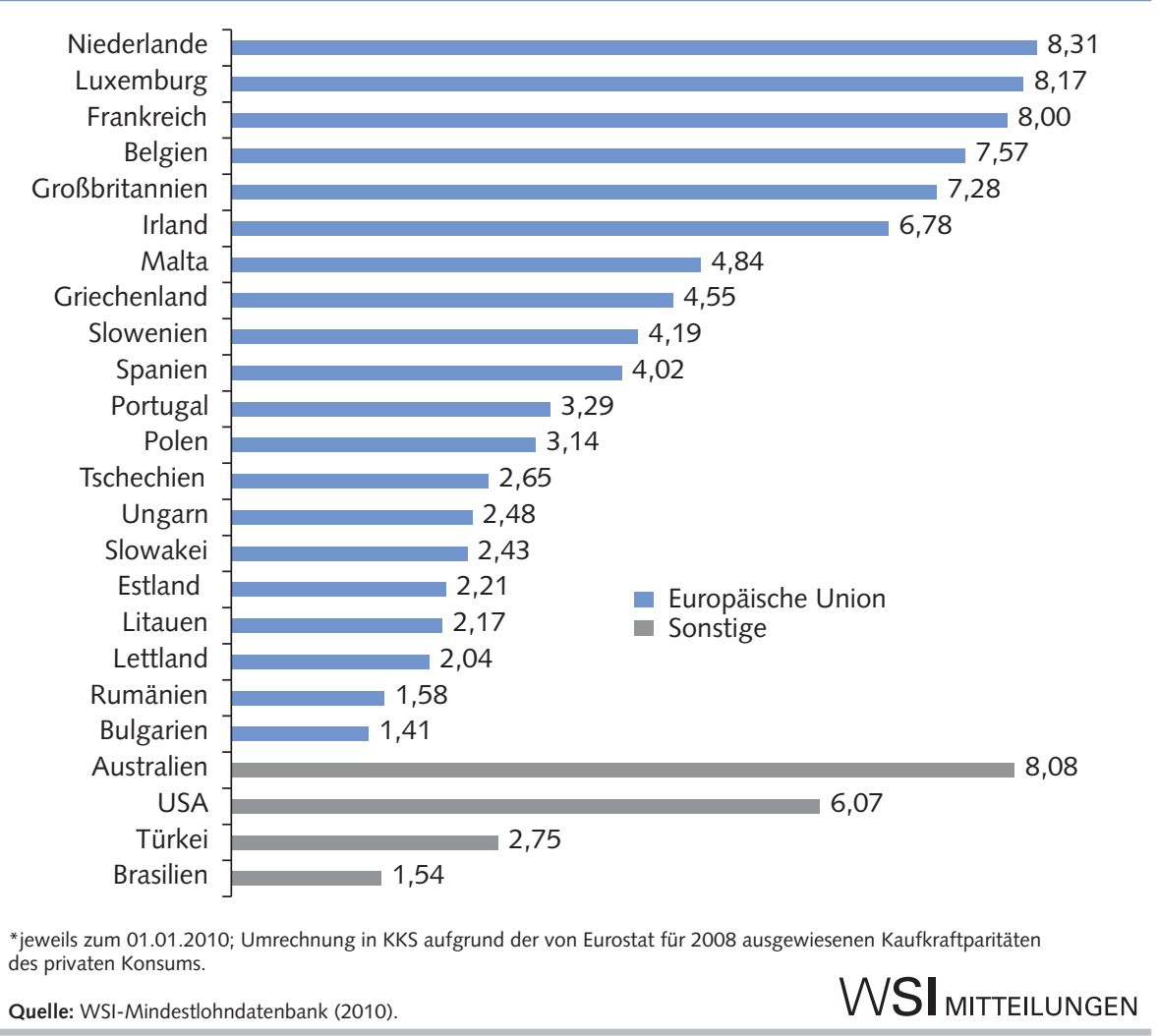

hen Mindestlöhnen gehören insgesamt sechs Staaten aus Westeuropa. Den absoluten Spitzenreiter bildet Luxemburg mit einem Mindestlohn von 9,73€ pro Stunde. In Belgien, Frankreich, Irland und den Niederlanden variieren die Mindeststundenlöhne (gerundet) zwischen $8,40 €$ und $9,00 €$. Etwas abgeschlagen von der Spitzengruppe liegt Großbritannien mit einem Mindestlohn von 6,51 € pro Stunde. Der Eurobetrag des britischen Mindestlohns wird jedoch stark vom Wechselkurs des britischen Pfundes beeinflusst, das in den letzten beiden Jahren gegenüber dem Euro um etwa $30 \%$ abgewertet wurde. Ohne diese Abwertung würde der britische Mindeststundenlohn heute bei etwa $8,50 €$ liegen und sich damit im Rahmen der anderen westeuropäischen Länder bewegen.

Eine zweite Gruppe mit Mindestlöhnen im europäischen Mittelfeld umfasst die südeuropäischen Länder Griechenland, Malta, Spanien und Portugal sowie Slowenien. In dieser Gruppe liegen die gesetzlichen Mindeststundenlöhne zwischen $2,68 €$ in Portugal und 4,28 $€$ in Griechenland. Zu der dritten Gruppe mit Mindestlöhnen unterhalb von $2 €$ gehören ausschließlich Länder aus Mittel- und Osteuropa. In den meisten Ländern dieser gerechnet 5,20€ und damit zwischen der west- und der südeuropäischen Gruppe. Allerdings existiert in 13 von 50 Bundesstaaten zusätzlich ein landesspezifischer gesetzlicher Mindestlohn, der oberhalb des nationalen Mindestlohniveaus liegt. Der US-Bundesstaat Washington verfügt dabei mit einem gesetzlichen Mindestlohn von umgerecht $6,12 €$ pro Stunde über den höchsten landesspezifischen Wert. In der Türkei befindet sich der gesetzliche Min- destlohn mit $1,73 €$ pro Stunde auf einem mit den meisten mittel- und osteuropäischen Staaten vergleichbaren Niveau. Der brasilianische Mindestlohn liegt schließlich mit $0,97 €$ pro Stunde bereits oberhalb des Betrages von Bulgarien und Rumänien.

Der Vergleich von gesetzlichen Mindestlöhnen in Euro wird jedoch nicht nur durch Wechselkursschwankungen verzerrt, er sagt auch nichts über den realen Wert des Mindestlohns aus, den dieser gemessen an den jeweiligen Lebenshaltungskosten für die betroffenen Beschäftigten hat. Um die Mindestlohnbeträge im Hinblick auf die unterschiedlichen nationalen Preisniveaus vergleichbar zu machen, müssen sie in Kaufkraftstandards (KKS) umgerechnet werden, wodurch sich das Gesamtbild erheblich verschiebt (Abbildung 2): Zunächst einmal nehmen die Niveauunterschiede zwischen den nationalen Mindestlöhnen deutlich ab: Während die Differenz zwischen dem höchsten und dem niedrigsten Mindestlohn gemessen in Euro bei etwa 1:14 liegt, beträgt sie gemessen in KKS lediglich 1:6.

Darüber hinaus kommt es durch die Berechnung in KKS auch zu erheblichen Veränderungen in der Rangfolge der Länder: Die Niederlande verfügen nun über den höchsten Mindestlohn in Europa. Luxemburg, das in Eurobeträgen den mit Abstand höchsten Mindestlohn aufweist, nimmt aufgrund des hohen Preisniveaus in dem Großherzogtum nur noch den zweiten Platz ein. Am deutlichsten reduziert sich der Wert des Mindestlohns bei der Umrechnung in KKS jedoch in Irland, das in der Länderrangfolge vom dritten auf den sechsten Platz zurückfällt. Im Gegensatz hierzu gewinnt der britische Mindestlohn gemessen in KKS deutlich an Wert. Einen sehr hohen Mindestlohnwert in KKS weist schließlich auch Australien auf, der in Europa lediglich von den Niederlanden und Luxemburg übertroffen wird. Insgesamt bleiben jedoch auch bei einer Berechnung in KKS erhebliche Niveauunterschiede bei den Mindestlöhnen bestehen, die das reale Lohngefälle zwischen den Staaten widerspiegeln.

\section{3 \\ Der relative Wert gesetz- licher Mindestlöhne}

Gesetzliche Mindestlöhne unterscheiden sich nicht nur aufgrund ihres absoluten Wertes, sondern auch im Hinblick auf ihre 
Stellung im jeweils nationalen Lohngefüge. Letztere kann durch den sogenannten Kaitz-Index bestimmt werden, der den relativen Wert des gesetzlichen Mindestlohns zum Ausdruck bringt und statistisch als Prozentsatz des Mindestlohns vom jeweiligen Durchschnitts- oder Medianlohn gemessen wird. ${ }^{3}$ Entsprechende Datensätze werden regelmäßig von der OECD und von EUROSTAT veröffentlicht. Aufgrund der methodischen Schwierigkeiten bei der Erhebung von Durchschnitts- und Medianlöhnen liegen derzeit jedoch lediglich Daten bis 2008 vor.

Der relative Wert des Mindestlohns weist insgesamt eine große Schwankungsbreite auf, die sich bezogen auf den Medianlohn zwischen $34 \%$ und $63 \%$ und bezogen auf den Durchschnittslohn zwischen $25 \%$ und $52 \%$ bewegt (Tabelle 1). Im Hinblick auf den Medianlohn liegt der relative Wert des Mindestlohns in der Mehrzahl der Länder zwischen $40 \%$ und $50 \%$. Ein vergleichsweise hohes Mindestlohnniveau mit Werten über $50 \%$ des Medianlohns findet sich in Australien, Belgien, Griechenland, Irland und Slowenien sowie in Frankreich, das mit knapp $63 \%$ des Medianlohns den höchsten relativen Mindestlohnwert aufweist. Extrem niedrige relative Mindestlohnwerte unterhalb von $40 \%$ des Medianlohns existieren in der Türkei, Tschechien und den USA, die mit $34 \%$ des Medianlohns das Schlusslicht bilden.

Im Hinblick auf den Durchschnittslohn bestehen zwischen den Datensätzen von OECD und EUROSTAT erhebliche Unterschiede, wobei EUROSTAT in der Regel höhere Werte aufweist, was damit zusammenhängen dürfte, dass die Grundeinheit der berücksichtigten Löhne bei der OECD gröBer ist und hierdurch auch die extrem hohen Lohneinkommen eher berücksichtigt werden. In der Mehrzahl der erfassten Länder bewegt sich der relative Wert des Mindestlohns zwischen $33 \%$ und $40 \%$. Lediglich vier Länder verfügen nach OECD-Daten über einen Mindestlohn, der über $40 \%$ des Durchschnittslohns liegt. Nach EUROSTAT-Daten sind es immerhin neun Länder. Der höchste relative Mindestlohn mit einem Wert um die $50 \%$ des Durchschnittslohns existiert nach OECD-Daten in Frankreich und nach EUROSTAT-Daten in Frankreich, Griechenland, Luxemburg und Malta. Am unteren Ende befinden sich wiederum die USA, deren relativer Mindestlohn nach OECD-Daten nur knapp

Tabelle 1: Der relative Wert des Mindestlohns 2000-2008 (Kaitz-Index)

\begin{tabular}{|c|c|c|c|c|c|c|c|c|c|}
\hline \multirow[t]{2}{*}{$\begin{array}{l}\text { Mindestlohn } \\
\text { in } \% \text { des ... }\end{array}$} & \multicolumn{3}{|c|}{$\begin{array}{l}\text { Medianlohns } \\
\text { (OECD) }\end{array}$} & \multicolumn{3}{|c|}{$\begin{array}{l}\text { Durchschnittslohns } \\
\text { (OECD) }\end{array}$} & \multicolumn{3}{|c|}{$\begin{array}{l}\text { Durchschnittslohns } \\
\text { in Industrie und } \\
\text { Dienstleistungen } \\
\text { (EUROSTAT) }\end{array}$} \\
\hline & 2000 & 2005 & 2008 & 2000 & 2005 & 2008 & 2002 & 2005 & 2008 \\
\hline Frankreich & 59,5 & 61,8 & 62,7 & 47,8 & 49,6 & 50,0 & & & $48,1^{* *}$ \\
\hline Griechenland & 47,1 & 48,3 & 53,1 & 36,7 & 37,6 & 41,3 & & & 49,3 \\
\hline Irland & 67,5 & 54,0 & 52,8 & 58,5 & 46,2 & 44,5 & 50,0 & 52,0 & 42,0 \\
\hline Australien & 58,2 & 57,5 & 52,2 & 50,1 & 49,5 & 45,0 & & & \\
\hline Belgien & 53,1 & 50,9 & 50,6 & 45,8 & 44,1 & 43,7 & 46,4 & & \\
\hline Slowenien & & 52,5 & 50,0 & & & & 45,3 & 45,6 & 43,6 \\
\hline Portugal & 47,4 & 48,2 & 47,1 & 33,4 & 34,0 & 33,2 & 43,0 & 40,5 & 39,9 \\
\hline Rumänien & 29,9 & 47,0 & 47,0 & 21,5 & 33,9 & 33,9 & 31,3 & 32,6 & 30,5 \\
\hline Ungarn & 37,2 & 48,1 & 46,6 & 28,2 & 36,1 & 34,6 & 42,1 & 38,2 & 36,7 \\
\hline Großbritannien & 40,8 & 45,0 & 46,1 & 34,1 & 37,0 & 38,0 & 34,4 & 37,0 & 37,4 \\
\hline Polen & 40,0 & 42,8 & 45,5 & 33,0 & 34,5 & 36,7 & 33,0 & 33,7 & 39,0 \\
\hline Spanien & 43,0 & 44,2 & 44,7 & 34,1 & 34,9 & 35,3 & 36,2 & 40,4 & 43,1 \\
\hline Slowakei & 42,1 & 43,2 & 43,0 & 33,7 & 33,9 & 32,8 & 32,4 & 34,4 & $34,8^{*}$ \\
\hline Niederlande & 47,1 & 44,4 & 42,9 & 41,7 & 39,0 & 37,6 & 49,3 & 45,5 & $44,2 * *$ \\
\hline Litauen & 47,7 & 49,3 & 42,1 & 37,3 & 39,3 & 33,9 & 36,9 & 38,3 & 35,2 \\
\hline Luxemburg & 39,8 & 41,5 & 40,5 & 32,7 & 34,0 & 33,2 & 50,4 & 50,7 & 50,2 \\
\hline Türkei & 25,1 & 37,3 & $36,1^{*}$ & 25,1 & $37,3^{*}$ & 36,1 & & & \\
\hline Tschechien & 31,7 & 38,7 & 35,3 & 27,3 & 33,2 & 30,0 & 36,9 & 39,1 & 35,0 \\
\hline USA & 35,8 & 31,6 & 34,1 & 28,5 & 24,5 & 25,4 & 34,4 & 31,9 & 34,0 \\
\hline Malta & & & & & & & 53,9 & 50,6 & 52,0 \\
\hline Bulgarien & & & & & & & 39,7 & 49,6 & 43,3 \\
\hline Lettland & & & & & & & 35,2 & 33,6 & 34,7 \\
\hline Estland & & & & & & & 30,5 & 33,2 & $30,5^{*}$ \\
\hline \multicolumn{7}{|l|}{$\begin{array}{l}{ }^{*} 2006 ;{ }^{*}{ }^{*} 2007 \\
\text { Quelle: Mindestlor }\end{array}$} & \multicolumn{3}{|c|}{ WSI mitteilungen } \\
\hline
\end{tabular}

über $25 \%$ liegt (nach EUROSTAT-Daten bei $34 \%)$.

Die gesetzlichen Mindestlöhne liegen insgesamt - zum Teil deutlich - unterhalb der von OECD und EUROSTAT verwendeten Niedriglohnschwelle, die bei zwei Dritteln des Medianlohns angesetzt wird. In Analogie zu der in der Armutsforschung verwendeten Armutsschwelle von $60 \%$ des Medianeinkommens oder 50 \% des Durchschnittseinkommens kann davon gesprochen werden, dass in vielen Ländern der gesetzliche Mindestlohn lediglich einen „Armutslohn“ festschreibt, der in der Regel nur ein sehr bescheidenes Einkommensniveau gewährleistet und in manchen Fällen sogar unterhalb des offiziellen Existenzminimums liegt. In vielen Ländern wird deshalb insbesondere von Seiten der Gewerkschaften und von anderen sozialen Organisationen eine strukturelle Erhöhung des gesetzlichen Mindestlohns gefordert, damit dieser ein bestimmtes sozio-kulturelles Existenzminimum ermöglicht und den Anforderungen eines „Living Wage“ entspricht. ${ }^{4}$

Die Veränderung des Kaitz-Index gibt schließlich darüber Auskunft, wie sich die Mindestlöhne im Vergleich zu den allgemeinen Lohnsteigerungen entwickelt ha- ben. Für den Zeitraum 2000-2008 zeichnen die hier untersuchten Staaten ein sehr uneinheitliches Bild (Tabelle 1): In einigen Ländern, zu denen Frankreich, Griechenland, Großbritannien, Polen und Spanien gehören, weist der Kaitz-Index einen eindeutig positiven Trend auf, sodass in diesen Ländern die Mindestlöhne stärker als die Median- und Durchschnittslöhne angestiegen sind. In anderen Ländern wie z. B. Australien, Belgien, Irland oder den Niederlanden ist der Trend hingegen eindeutig negativ, womit die Mindestlöhne hinter der allgemeinen Lohnentwicklung zurückblieben. In einer dritten Gruppe, zu der vor allem Länder aus Mittel- und Osteuropa gehören, nahm der Kaitz-Index in der ersten Hälfte der letzten Dekade einen po-

3 Der Kaitz-Index wird nach dem amerikanischen Ökonomen Hyman Kaitz benannt (vgl. Kaitz 1970). Während der Durchschnittslohn durch das arithmetische Mittel aller Löhne bestimmt wird, ist der Medianlohn der mittlere, bei dem die Hälfte aller Beschäftigten mehr und die andere Hälfte weniger verdient.

4 Für den im Englischen etablierten Begriff „living wage" gibt es keine direkte deutsche Entsprechung. Wörtlich bedeute er "einen Lohn, von dem man leben kann", wobei hier mit "leben" in der Regel ein breit gefasstes sozio-kulturelles Existenzminimum gemeint ist. 


\begin{tabular}{|c|c|c|}
\hline \multicolumn{3}{|c|}{$\begin{array}{l}\text { Abb. 3: Nominale Entwicklung gesetzlicher Mindestlöhne 2009* } \\
- \text { in \% - }\end{array}$} \\
\hline & & zuletzt erhöht: \\
\hline Brasilien ] & 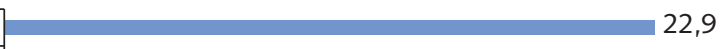 & 01.01 .2010 \\
\hline USA & 10,7 & 24.07.2009 \\
\hline Türkei & 9,5 & 01.01 .2010 \\
\hline Griechenland & 5,6 & 01.05 .2009 \\
\hline Portugal & 5,6 & 01.01 .2010 \\
\hline Slowakei & 4,2 & 01.01 .2010 \\
\hline Malta & 4,0 & 01.01 .2010 \\
\hline Polen & 3,2 & 01.01 .2010 \\
\hline Ungarn & 2,8 & 01.01 .2010 \\
\hline Luxemburg & 2,5 & 01.03 .2009 \\
\hline Niederlande - & 2,0 & 01.01 .2010 \\
\hline Frankreich & 1,7 & 01.01 .2010 \\
\hline Spanien & 1,5 & 01.01 .2010 \\
\hline Slowenien & 1,3 & 01.08 .2009 \\
\hline Großbritannien ] & 1,2 & 01.10 .2009 \\
\hline Australien & 0,0 & 01.10 .2008 \\
\hline Belgien & 0,0 & 01.10 .2008 \\
\hline Bulgarien & 0,0 & 01.01 .2009 \\
\hline Estland & 0,0 & 01.01 .2008 \\
\hline Irland & 0,0 & 01.07 .2007 \\
\hline Lettland & 0,0 & 01.01 .2009 \\
\hline Litauen & 0,0 & 01.01 .2008 \\
\hline Rumänien & 0,0 & 01.01 .2009 \\
\hline Tschechien ] & 0,0 & 01.01 .2007 \\
\hline \multicolumn{3}{|c|}{$\begin{array}{l}\text { *Entwicklung vom 01.01.2009 zum 01.01.2010. } \\
\text { Quelle: WSI-Mindestlohndatenbank (2010). }\end{array}$} \\
\hline
\end{tabular}

sitiven Verlauf, während sich der Trend in den letzten Jahren wieder ins Negative umgekehrt hat. Die bereits für 2008 konstatierte Trendwende mit erheblich niedrigeren Zuwachsraten bei den gesetzlichen Mindestlöhnen (Schulten 2009) hat sich im Jahr 2009 noch einmal deutlich verstärkt.

\section{$\angle$ \\ Die aktuelle Entwicklung der Mindestlöhne im Jahr 2009}

\section{1 ÜBERBLICK}

Die Mindestlohnpolitik im Jahr 2009 stand vollständig unter dem Vorzeichen der globalen Wirtschaftskrise, die in den einzelnen Ländern zu sehr unterschiedlichen Reaktionen geführt hat (Abbildung 3). Zwar wurden in keinem Land die Mindestlöhne nominal gekürzt; mehr als ein Drittel der hier untersuchten Staaten (darunter acht EU-Staaten plus Australien) haben ihre Mindestlöhne 2009 jedoch eingefroren. Demgegenüber haben zehn Länder (darunter acht EU-Staaten plus Brasilien und die Türkei) ihre Mindestlöhne zuletzt zum ke Rückgang der Verbraucherpreise dazu geführt, das trotz eines eingefrorenen nominalen Mindestlohnniveaus der reale Mindestlohnwert weiter angestiegen ist. Die negative Preisentwicklung hat auch in Portugal dazu beigetragen, dass der bereits hohe nominale Mindestlohnzuwachs real noch einmal um einen Prozentpunkt übertroffen wurde. Portugal konnte somit 2009 mit 6,5 \% den höchsten Reallohnanstieg in der EU verzeichnen. Übertroffen wurde dieser lediglich von den USA und Brasilien, wo der reale Wert des gesetzlichen Mindestlohns um 11,3\% bzw. 18,1 \% zunahm.

\subsection{BENELUX-STAATEN UND FRANKREICH}

Die BeNeLux-Staaten und Frankreich gehören (gemeinsam mit Malta) zu denjenigen Ländern, in denen eine automatische Indexierung der Mindestlöhne existiert. Dies bedeutet, dass die Mindestlöhne automatisch an die Preissteigerungsrate (in Belgien und Luxemburg), die durchschnittliche Entwicklung der Tariflöhne (in den Niederlanden) oder die Preise plus die Hälfte des durchschnittlichen Lohnanstiegs (in Frankreich) angepasst werden. Darüber hinaus sind in allen Ländern auch zusätzlich „politische“ Anpassungen der Mindestlohnsätze möglich.

2009 haben sich die Erhöhungen der Mindestlöhne jedoch in allen Ländern auf das gesetzlich vorgeschriebene Mindestmaß beschränkt. In Belgien hat die sehr niedrige Steigerung der Verbraucherpreise dazu geführt, dass seit der letzten Erhöhung des Mindestlohns im Oktober 2008 keine neue Anpassung mehr vorgenommen und damit das Mindestlohnniveau 2009 faktisch eingefroren wurde. In $\mathrm{Lu}$ xemburg ist der gesetzliche Mindestlohn zuletzt im März 2009 im Rahmen der automatischen Indexierung der Löhne an die Preissteigerungsrate um 2,5 \% erhöht worden. In den Niederlanden, wo die Mindestlöhne traditionell zweimal jährlich angepasst werden, summieren sich die beiden Erhöhungen zum 1. Juli 2009 und zum 1. Januar 2010 auf insgesamt 1,9\% und entsprechen damit lediglich den gesetzlichen Mindestanforderungen.

Während die Anpassung der Mindestlöhne in den BeNeLux-Staaten ohne gröBere öffentliche Debatte verlief, war in Frankreich der SMIC („Salaire minimum interprofessionel de croissance") Gegenstand kontroverser politischer Auseinan- 
dersetzungen. Die konservative Regierung unter Nicolas Sarkozy hatte bereits 2008 die Absicht verkündet, das französische Mindestlohnsystem zu reformieren. Den Hintergrund hierfür bildete die vor allem von den Arbeitgeberverbänden vertretene Auffassung, wonach der französische Mindestlohn mittlerweile ein zu hohes Niveau erreicht hätte und sich negativ auf die Beschäftigung auswirken würde. In zwei eigens von der französischen Regierung hierzu beauftragten Gutachten wurde diese Einschätzung bestätigt und als zentrales Reformvorhaben die Abschaffung der automatischen Indexierung des Mindestlohns an die Preis- und Lohnentwicklung gefordert (Cahuc et al. 2008; COE 2008).

Aufgrund des starken politischen Widerstandes hat sich die französische Regierung diese Position jedoch nicht zu eigen gemacht, sondern sich im Rahmen einer im Dezember 2008 verabschiedeten Gesetzesreform auf einige neue Verfahrensregeln beschränkt. Hierzu gehört zum einen, dass die jährliche Anpassung des Mindestlohns nicht mehr wie bislang zum 1.7., sondern zum 1.1. ein Jahres erfolgt, um auf diese Weise es den Tarifvertragsparteien zu erleichtern, in den jährlichen Verhandlungen die neuen Mindestlohnsätze zu berücksichtigen. Zum anderen wurde 2009 eine Expertenkommission (Groupe d'Experts sur le SMIC) mit fünf Wissenschaftlerinnen und Wissenschaftlern eingerichtet, die die Regierung in ihrer Mindestlohnpolitik beraten soll.

Die Anpassung des gesetzlichen Mindestlohns zum 1. Juli 2009 und zum 1. Januar 2010 erfolgte erstmals auf der Grundlage einer von der Expertenkommission ausgesprochenen Empfehlung, in der sie sich einmütig dafür aussprach, die Erhöhung des Mindestlohns auf das gesetzlich vorgeschriebene Mindestmaß zu beschränken (Groupe d'Experts sur le SMIC 2009a, 2009b). Da alle Kommissionsmitglieder eher aus einer neoklassischen Theorietradition kommen, ist es nicht sehr verwunderlich, dass die Empfehlung für eine möglichst geringe Mindestlohnerhöhung mit der Notwendigkeit begründet wurde, angesichts des schwierigen wirtschaftlichen Umfeldes den Verlust weiterer Arbeitsplätze zu vermeiden.

Mit einer Zunahme von insgesamt 1,7 \% verzeichnet der französische Mindestlohn 2009 die geringste nominale Steigerungsrate seit mehr als zehn Jahren. Allerdings entspricht die Mindestlohner-

Abb. 4: Reale Entwicklung gesetzlicher Mindestlöhne 2009* - in $\%-$

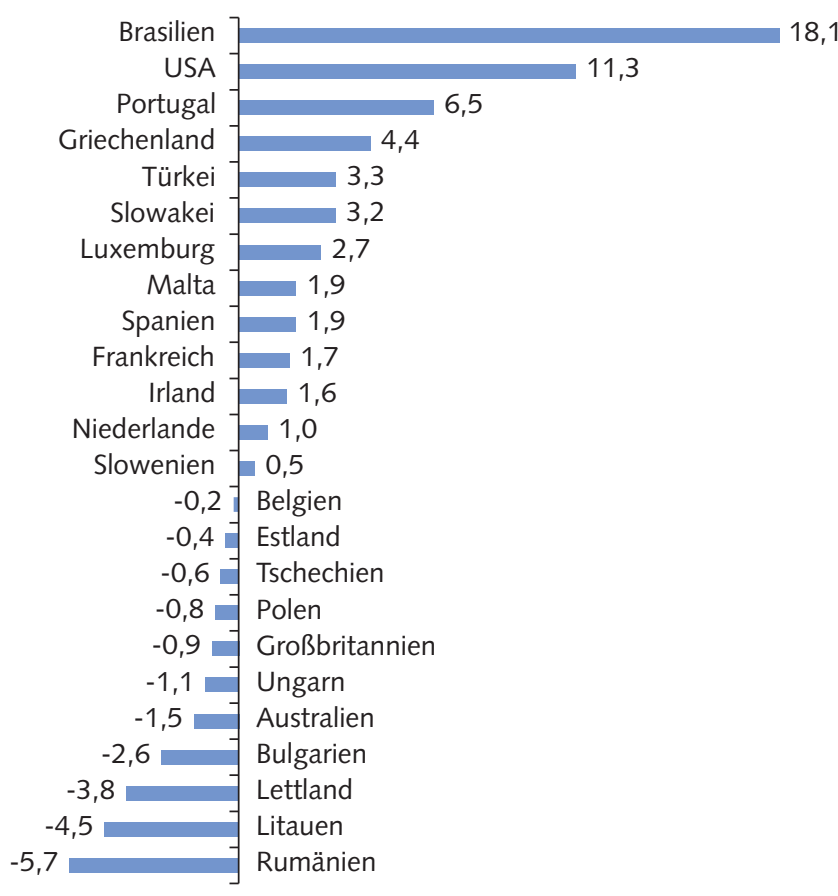

*Entwicklung vom 01.01.2009 zum 01.01.2010 in \%, bereinigt um die Entwicklung der Verbraucherpreise.

höhung aufgrund der stagnierenden Preisentwicklung einer entsprechenden Erhöhung des realen Mindestlohnwertes, die deutlich höher als in den Vorjahren ausfällt. Trotzdem haben die linken französischen Oppositionsparteien und die französischen Gewerkschaften die Mindestlohnpolitik der Sarkozy-Regierung scharf kritisiert und eine deutlich stärkere Erhöhung des Mindestlohns gefordert (Le Monde, 15.12.2009). Der größte französische Gewerkschaftsverband CGT hat hierbei seine Forderung nach einer strukturellen Anhebung des Mindestlohns auf $1.600 €$ pro Monat bekräftigt (Dumas 2009), was bei der in Frankreich geltenden Regelarbeitszeit von 35 Stunden pro Woche einem Mindestlohn von etwa 10,50€ pro Stunde entsprechen würde.

\subsection{GROßBRITANNIEN UND IRLAND}

Sowohl Großbritannien als auch Irland verfügen erst seit Beginn der Jahrtausendwende über einen nationalen, branchenübergreifenden gesetzlichen Mindestlohn. Dieser nahm in beiden Ländern jedoch einen sehr unterschiedlichen Verlauf. Während Großbritannien mit einem relativ niedrigen Mindestlohn einstieg und diesen seither kontinuierlich erhöht hat, hat Irland ursprünglich mit einem deutlich höheren Mindestlohnniveau begonnen, das zudem in der Mitte des letzten Jahrzehnts noch einmal stark angehoben wurde. Seit Juli 2007 ist der irische Mindestlohn jedoch auf einem Niveau von $8,65 €$ pro Stunde eingefroren.

In Großbritannien wird der Mindestlohn regelmäßig zum 1. Oktober eines Jahres angepasst. Bei der Entscheidung über die Höhe des Mindestlohnanstiegs ist die britische Regierung bislang stets der Empfehlung der sogenannten Low Pay Commission gefolgt, die sich jeweils zu einem Drittel aus Vertretern von Arbeitgebern, Gewerkschaften und Wissenschaft zusammensetzt. Die Low Pay Commission führt regelmäßig umfangreiche Anhörungen durch und gibt eigene Studien in Auftrag, deren Ergebnisse in einem jährlichen Report zusammengefasst werden, auf dessen Grundlage sie dann ihre Empfehlung ausspricht (zuletzt: Low Pay Commission 2009).

Für das Jahr 2009 hat die Low Pay Commission sich angesichts der Wirtschaftskrise lediglich für einen sehr moderaten Anstieg des Mindestlohns um 1,2 \% auf 5,80 Pfund ausgesprochen. Hierbei handelt es sich um den geringsten Anstieg 
seit Einführung des gesetzlichen Mindestlohns, was dazu geführt hat, dass der reale Wert des Mindestlohns gesunken ist. Im Vorfeld hatten sich die britischen Arbeitgeber lediglich für einen sehr geringen Anstieg ausgesprochen oder sogar offen ein Einfrieren des Mindestlohnniveaus gefordert (ebd., S. 227ff.). Im Gegensatz hierzu haben die britischen Gewerkschaften für eine deutliche Erhöhung des Mindestlohns votiert, um angesichts der Krise den privaten Konsum zu stärken (ebd., S. 230ff.). Der britische Gewerkschaftsdachverband TUC hat für 2009 eine Erhöhung um 6,5 \% auf 6,10 Pfund und für 2010 um weitere 6,5\% auf 6,50 Pfund gefordert. Einzelne Gewerkschaften innerhalb des TUC haben hingegen noch einmal für eine deutlich höhere Steigerung des Mindestlohns plädiert, um den Mindestlohn strukturell auf das Niveau eines „living wage“ anzuheben. So fordert z. B. die Public and Commercial Services Union einen gesetzlichen Mindestlohn, der sich an der Niedriglohnschwelle von zwei Dritteln des nationalen Medianlohns orientiert und nach Gewerkschaftsangaben derzeit bei etwa 8,25 Pfund liegt.

Im Gegensatz zu Großbritannien ist in Irland der gesetzliche Mindestlohn bereits im zweiten Jahr in Folge nicht mehr angehoben worden. Angesichts der Weltwirtschaftskrise, die in Irland stärker als in jedem anderen westeuropäischen EU-Staat zu einem gewaltigen Rückgang der Wirtschaftsleistung geführt hat, ist dort eine intensive Debatte über eine mögliche Kürzung des nominalen Mindestlohnsatzes entbrannt. Eine entsprechende Forderung ist nicht nur von mehreren Arbeitgeberverbänden erhoben worden, sie wurde indirekt auch von einzelnen Mitgliedern der irischen Regierung unterstützt (Irish Times, 11.2. 2009). Unter der Überschrift "Cutting minimum wage is on the table" berichtet die irische Tageszeitung Independent (vom 21.7. 2009) darüber, dass einzelne einflussreiche irische Ökonomen die Forderung nach Lohnkürzungen dahingehend konkretisiert haben, dass zum Ausgleich für die verloren gegangene Wettbewerbfähigkeit der irischen Industrie das Lohnniveau insgesamt um $10 \%$ bis $15 \%$ reduziert werden müsste und auch der Mindestlohn hiervon nicht ausgenommen werden dürfe.

Während sich die irischen Gewerkschaften noch Ende 2008 für eine deutliche Erhöhung des irischen Mindestlohns einsetzten (Irish Times, 16.12. 2008), konzen- trierten sie sich im Laufe des Jahres 2009 immer mehr darauf, eine Kürzung des Mindestlohns zu verhindern. Nach Ansicht des Chefökonomen des irischen Gewerkschaftsbundes Paul Sweeney würde eine Kürzung der Mindestlöhne die Krise eher noch verschärfen, da sie die Binnennachfrage weiter reduzieren und die gesamte Wirtschaft weiter in eine Deflationsspirale drängen würde (Sweeney 2009). In der Tat lässt sich in keinem anderen europäischen Land ein so starker Rückgang der Verbraucherpreise - nämlich um 1,6 \% im Jahr 2009 - beobachten wie in Irland. Diese Entwicklung hat eindeutig eine deflationäre Tendenz.

\subsection{SPANIEN UND PORTUGAL}

Sowohl in Spanien als auch in Portugal verfolgen die jeweils von sozialistischen Parteien geführten Regierungen erklärtermaßen das Ziel, das Niveau des gesetzlichen Mindestlohns langfristig deutlich anzuheben. In Spanien haben sich die Zapatero-Regierung und die spanischen Gewerkschaften unter Bezugnahme auf die Europäische Sozialcharta des Europarates darauf verständigt, dass der spanische Mindestlohn langfristig bei $60 \%$ des Durchschnittslohns liegen soll. Mit dem Antritt ihrer zweiten Amtszeit im Jahr 2008 formulierte die Regierung das konkrete Ziel, den Mindestlohn bis zum Ende der aktuellen Legislaturperiode im Jahr 2012 von damals $600 €$ pro Monat $(3,64 €$ pro Stunde) auf $800 €$ pro Monat ( 4,85 pro Stunde) zu erhöhen. Nachdem in den letzten Jahren der Mindestlohn jeweils zwischen $4 \%$ und 5,5\% angestiegen ist, fiel die Erhöhung zum 1. Januar 2010 mit lediglich 1,5 \% eher bescheiden aus. Die Regierung rechtfertigte den geringen Anstieg des Mindestlohns auf $3,84 €$ pro Stunde mit den schwierigen ökonomischen Rahmenbedingungen in der Krise und dem rasanten Anstieg der Arbeitslosigkeit (Ministerio de Trabajo e Immigacion 2009). Die spanischen Gewerkschaften haben den geringen Mindestlohnzuwachs scharf kritisiert und darauf hingewiesen, dass die Krise nicht als Ausrede benutzt werden dürfe, um die strukturelle Erhöhung des Mindestlohns aufzugeben. Um das Ziel von $800 €$ pro Monat nicht aus den Augen zu verlieren, forderten die Gewerkschaften eine Erhöhung des Mindestlohns um $8 \%$ auf $674 €$ pro Monat $(4,08 €$ pro Stunde) (El Pais, 22.12. 2009).
In Portugal wurde im Dezember 2006 ein tripartistisches Abkommen zwischen der sozialistischen Regierung unter José Sócrates, den Arbeitgeberverbänden und den Gewerkschaften abgeschlossen, demzufolge der gesetzliche Mindestlohn zwischen 2007 und 2011 um jahresdurchschnittlich $5,3 \%$ auf $500 €$ pro Monat $(3,01 €$ pro Stunde) ansteigen soll (da Paz et al. 2006). Anders als in Spanien hat die portugiesische Regierung trotz der Wirtschaftskrise und gegen den Widerstand der portugiesischen Arbeitgeberverbände an dem langfristigen Abkommen festgehalten und den Mindestlohn zum 1. Januar 2010 um 5,6 \% erhöht. Angesicht eines Rückgangs der Verbraucherpreise von knapp $1 \%$ stieg der reale Wert des portugiesischen Mindestlohns sogar um 6,5\% und damit schneller als in jedem andern europäischen Land.

\subsection{DIE STAATEN IN MITTEL- UND OSTEUROPA}

Vor dem Hintergrund einer vergleichsweise schwach entwickelten Tarifstruktur mit einer relativ niedrigen Tarifbindung hat der gesetzliche Mindestlohn für die Lohnentwicklung in vielen mittel- und osteuropäischen Ländern eine zentrale Bedeutung. Nachdem im letzten Jahrzehnt die Mindestlöhne in vielen dieser Länder kräftig angehoben wurden, wurden sie 2009 angesichts der Wirtschaftskrise in der Mehrzahl der Staaten eingefroren. Lediglich in Polen, der Slowakei, Slowenien und Ungarn kam es zu einem nominalen Anstieg der Mindestlöhne. Der um die Inflationsrate bereinigte reale Wert des Mindestlohns nahm hingegen lediglich in der Slowakei und in Slowenien zu. Die große Mehrheit der Mindestlohnbezieher in Mittel- und Osteuropa hatte demnach 2009 erhebliche Kaufkraftverluste zu verzeichnen.

Einen besonders dramatischen Wirtschaftseinbruch erlebten 2009 die baltischen Staaten, was dazu führte, dass in diesen Ländern offen über nominale Lohnkürzungen diskutiert wird. In Lettland hatte die dortige Regierung bereits einen Gesetzesentwurf vorgelegt, der eine Kürzung des gesetzlichen Mindestlohns um $22 \%$ vorsah und damit deutlich unterhalb des offiziellen Existenzminimums gelegen hätte. Nachdem nicht nur die lettischen Gewerkschaften, sondern auch Teile der lettischen Arbeitgeber sich gegen eine nominale Mindestlohnkürzung ausgespro- 
chen haben, hat die Regierung schließlich von ihren Plänen Abstand genommen (SOLIDAR 2009, S. 21).

In vielen mittel- und osteuropäischen Ländern wird der nationale Mindestlohn im Rahmen tripartistischer Vereinbarungen zwischen Regierung, Arbeitgebern und Gewerkschaften festgelegt. In Polen hatte sich die Trilaterale Kommission bereits im Juli 2009 auf eine Erhöhung des gesetzlichen Mindestlohns um 3,2 \% verständigt. In Ungarn hat der Nationale Schlichtungsrat (OET) eine Erhöhung des Mindestlohns um 2,8 \% beschlossen, nachdem die ungarischen Gewerkschaften zuvor eine deutlich kräftigere Erhöhung von $9 \%$ gefordert hatten, da der derzeitige Mindestlohn unterhalb des offiziellen Existenzminimums liegt.

Keine Verständigung über die Erhöhung des Mindestlohns haben Arbeitgeber und Gewerkschaften in der Slowakei erzielen können. Während die Arbeitgeber für ein Einfrieren des slowakischen Mindestlohns eintraten, forderten die Gewerkschaften eine Erhöhung um $8 \%$. Die sozialdemokratische Regierung hatte ursprünglich die Forderung der Gewerkschaften unterstützt, sich letztlich jedoch für eine erheblich geringere Erhöhung von $4,2 \%$ entschieden. Damit beschert die Slowakei jedoch immer noch als einziges osteuropäisches Land seinen Mindestlohnbeziehern einen kräftigen Reallohnzuwachs. Eine äußerst kontroverse Auseinandersetzung um den Mindestlohn wird auch in Slowenien geführt. Die sozialdemokratisch geführte Regierung hatte zum 1. August 2009 eine geringe Erhöhung um 1,3\% auf 3,45€ pro Stunde beschlossen. Um ein angemessenes Existenzminimum in Slowenien zu gewährleisten, fordern die slowenischen Gewerkschaften dagegen einen Netto-Mindestlohn von $600 €$ pro Monat, der brutto einem Mindestlohn von etwa $830 €$ pro Monat $(4,80 €$ pro Stunde) entsprechen würde. Für eine hierfür notwendige strukturelle Erhöhung des Mindestlohns um $40 \%$ haben die slowenischen Gewerkschaften im November 2009 ein große Demonstration mit etwa 30.000 Teilnehmern organisiert (Slovenian Press Agency, 28.11.2009). Schließlich legte die slowenische Regierung Ende Januar 2010 einen neuen Gesetzentwurf vor, der für 2010 eine substanzielle Erhöhung des Mindestlohns um $23 \%$ auf $734 €$ pro Monat $(4,24 €$ pro Stunde) vorsieht.
In Rumänien hatten sich Regierung, Arbeitgeber und Gewerkschaften zunächst im Herbst 2009 auf eine Erhöhung des Mindestlohns um $17,5 \%$ verständigt. Teile der rumänischen Arbeitgeber haben sich jedoch anschließend massiv für ein Einfrieren des Mindestlohnniveaus stark gemacht. Die im Dezember 2009 neu gewählte liberal-konservative Regierung hat die Entscheidung über eine Mindestlohnerhöhung erst einmal verschoben und damit de facto der Forderung des Arbeitgeberlagers nachgegeben. In Bulgarien haben sich im Nationalen Tripartistischen Rat Regierung und Arbeitgeber gegen das Votum der Gewerkschaften ebenfalls für ein Einfrieren des Mindestlohns ausgesprochen. Schließlich hat Tschechien als einziges Land in Europa bereits im dritten Jahr in Folge keine Erhöhung des Mindestlohns vorgenommen. Vor dem Hintergrund der Wirtschaftskrise hatten sich die tschechischen Gewerkschaften darauf beschränkt, einen Inflationsausgleich für den Mindestlohn zu fordern.

\subsection{EUROPÄISCHE LÄNDER OHNE GESETZLICHEN MINDESTLOHN}

In sieben EU-Staaten (darunter die skandinavischen Länder Dänemark, Finnland und Schweden sowie Italien, Zypern, Österreich und Deutschland) existiert kein allgemeiner, branchenübergreifender gesetzlicher Mindestlohn. Das gleiche gilt außerhalb der EU auch für Island, Norwegen und die Schweiz. In diesen Ländern werden Mindestlöhne ausschließlich durch Tarifverträge geregelt, sodass die Mindestlohnsicherung im Wesentlichen von der Tarifbindung abhängt. Da Letztere in vielen dieser Länder relativ hoch ist (Schulten 2010), ist die Einführung eines gesetzlichen Mindestlohns dort derzeit kein Thema und wird auch von den jeweiligen Gewerkschaften nicht gewünscht.

Anders sieht es in Deutschland aus, wo eine abnehmende Tarifbindung und eine deutliche Ausdehnung des Niedriglohnsektors zu einem grundlegenden Meinungsumschwung bei den deutschen Gewerkschaften geführt haben und die Forderung nach Einführung eines gesetzlichen Mindestlohns auf der Tagesordnung steht. In der Schweiz haben die dortigen Gewerkschaften über Jahre hinweg eine erfolgreiche tarifpolitische Mindestlohnkampagne organisiert (Schulten 2008a). Aufgrund der fehlenden Tarifbindung ist dieser Ansatz jedoch in einigen Bereichen an Grenzen gestoßen. Der Schweizerische Gewerkschaftsbund hat deshalb im November 2009 einen Grundsatzbeschluss gefasst, im Jahr 2010 eine Mindestlohn-Volksinitiative zur Einführung eines gesetzlichen Mindestlohns in der Schweiz durchzuführen (Neue Züricher Zeitung, 9.11.2009).

Vor dem Hintergrund eines nach wie vor sehr hohen gewerkschaftlichen Organisationsgrades und einer hohen Tarifbindung werden gesetzliche Mindestlöhne besonders von den skandinavischen Gewerkschaften mit großer Skepsis betrachtet, da sie als Gefahr für die gewerkschaftliche Tarifautonomie angesehen werden. Allerdings hat insbesondere die zunehmende Arbeitsmigration nach der EU-Osterweiterung auch in den skandinavischen Ländern tarifpolitische Regelungslücken zum Vorschein gebracht. Hinzu kommt, dass durch jüngste Urteile des Europäischen Gerichtshofes bestimmte Praktiken der skandinavischen Gewerkschaften zur Erzwingung von Tarifverträgen als Verstoß gegen die europäische Dienstleistungs- und Niederlassungsfreiheit gewertet werden. Vor diesem Hintergrund hat auch in den skandinavischen Ländern eine kontroverse Debatte über gesetzliche Mindestlöhne begonnen. In Finnland hat der finnische Gewerkschaftsdachverband SAK im Sommer 2009 die Forderung nach einem allgemeinen Mindestlohn von $1.500 €$ pro Monat erhoben (Jokivuori 2009), der bei einer durchschnittlichen tariflichen Wochenarbeitszeit von 37,5 Stunden einem Mindeststundenlohn von 9,20€ entsprechen würde. Der allgemeine Mindestlohn soll nach Vorstellungen des Gewerkschaftsverbandes zunächst auf tarifpolitischem Wege durchgesetzt werden. Einen ähnlichen Weg ist im Jahr 2007 auch Österreich gegangen, wo die Dachverbände von Arbeitgebern und Gewerkschaften in einer Rahmenvereinbarung beschlossen haben, dass in allen Tarifverträgen die unterste Tarifgruppe nicht unter $1.000 €$ pro Monat liegen darf, was bei einer durchschnittlichen tariflichen Wochenarbeitszeit von 39 Stunden einem Mindeststundenlohn von 5,92 € entspricht.

\subsection{AUSTRALIEN, BRASILIEN UND DIE USA}

In Australien wird der gesetzliche Mindestlohn durch eine eigene Mindestlohnkommission festgelegt, deren Ergebnis jedoch anders als in Großbritannien für die Regie- 
rung einen bindenden Charakter hat. In den letzten Jahren wurde diese Aufgabe von der Australian Fair Pay Commission wahrgenommen, die sich aus fünf Wissenschaftlerinnen und Wissenschaftlern zusammensetzte. Zukünftig wird diese Aufgabe wieder ein politisches Mindestlohngremium übernehmen, das aus Vertretern des australischen Parlaments besteht. Für das Jahr 2009 hatte die Australian Fair Pay Commission (2009) im Hinblick auf die Wirtschaftskrise beschlossen, den Mindestlohn einzufrieren. Die australischen Gewerkschaften haben hingegen mit einer ausführlichen Analyse der ökonomischen Lage ihre Forderung nach einer Mindestlohnerhöhung von knapp $4 \%$ begründet (ACTU 2009).

In Brasilien kommt dem gesetzlichen Mindestlohn für die Einkommensentwicklung breiter Bevölkerungsschichten eine zentrale Rolle zu. Dies gilt nicht nur für die relativ hohe Anzahl von offiziellen Mindestlohnbeziehern, sondern auch für die zahlreichen Beschäftigten in der informellen Ökonomie, wo der Mindestlohn eine zentrale Referenzgröße bildet. Hinzu kommt, dass der Mindestlohn auch das Einkommen von Millionen von Rentnern beeinflusst, deren Renten an die Mindestlohnentwicklung gekoppelt sind. Seit dem Amtsantritt der sozialistischen Regierung unter Präsident Lula da Silva hat die Mindestlohnpolitik in Brasilien stark an Bedeutung gewonnen. So ist der reale Wert des Mindestlohns seit 2003 um mehr als $50 \%$ angestiegen.

Unter den Bedingungen der Krise verfolgt die brasilianische Regierung eine besonders expansive Mindestlohnpolitik. Zum 1. Februar 2009 erhöhte sie den Mindestlohn zunächst um $12 \%$ und zum 1 . Januar 2010 noch einmal um knapp $10 \%$, sodass sich insgesamt für das Jahr 2009 ein nominaler Zuwachs von $22 \%$ ergibt, der nach Abzug der Preissteigerungsrate real immer noch bei 18 \% liegt. Der brasilianischen Regierung ist es damit erfolgreich gelungen, einen erheblichen Nachfrageschub gegen die Krise zu organisieren.
Die USA zeichnen sich schließlich bereits seit Langem durch eine sehr unstetige Mindestlohnpolitik aus, bei der sich längere Phasen stagnierender Mindestlöhne mit kurzen Phasen relativ hoher Mindestlohnerhöhungen abwechseln. Nachdem der amerikanische Mindestlohn zehn Jahre lang nicht mehr angehoben wurde, wurde 2007 noch unter der Bush-Administration eine Erhöhung von $40 \%$ beschlossen, die zwischen 2007 und 2009 in drei Stufen erfolgen sollte. Im Sommer 2009 wurde von einigen amerikanischen Ökonomen, wie z. B. dem bekannten Mindestlohnkritiker David Neumark (2009), erfolglos die Forderung erhoben, angesichts der Krise die letzte Stufe der Mindestlohnerhöhung von etwas mehr als $10 \%$ auszusetzen. Andere Ökonomen haben dagegen darauf verwiesen, dass die beschlossenen Mindestlohnerhöhung gerade unter den Bedingungen der Krise besonders wichtig ist, da sie zu einer starken Erhöhung des privaten Konsums führt (Filion 2009).

\section{5 \\ Fazit: Alternative Mindest- lohnpolitiken in der Krise}

Die sehr unterschiedliche Entwicklung gesetzlicher Mindestlöhne in Europa und einigen hier ausgewählten außereuropäischen Ländern macht deutlich, dass es auch unter den Bedingungen der Krise alternative politische Handlungsmöglichkeiten gibt. Auf der einen Seite stehen diejenigen Länder, die der Krise dadurch zu entgehen versuchen, dass sie ihre Kosten möglichst gering halten. Unter dem politischen Druck der Arbeitgeberverbände und mit wissenschaftlicher Unterstützung orthodoxer Ökonomen haben sie ihre Mindestlöhne zumeist eingefroren oder nur geringfügig erhöht. In einigen Ländern wird sogar die Möglichkeit einer nominellen Mindestlohnkürzung diskutiert - auch wenn dies in der Praxis bislang nicht durchgesetzt werden konnte.
Der amerikanische Ökonom und Nobelpreisträger Paul Krugman (2009) hat entsprechende Forderungen in den USA als eine "totally counterproductive idea" bezeichnet. Eine Kürzung der Mindestlöhne würde Krugman zufolge die Krise eher noch verschlimmern, da sie die private Nachfrage drosselt und insgesamt eine deflationäre Entwicklung befördert. Innerhalb Europas sind die Gefahren einer solchen Entwicklung schon heute vor allem in Irland zu beobachten, das sich mittlerweile eindeutig auf einem ökonomischen Deflationspfad befindet.

Die Internationale Arbeitsorganisation ILO (2009) hat in ihrem Mitte 2009 verabschiedeten "Global Jobs Pact" auf die zentrale Rolle von Mindestlöhnen hingewiesen, um eine mögliche deflationäre Lohn-Preis-Spirale zu verhindern. Dem Mindestlohn kommt hierbei deshalb eine besonders wichtige Funktion zu, da er in vielen Ländern nicht nur das unmittelbare Lohneinkommen der Niedriglohnbezieher beeinflusst, sondern als Orientierungsmarke für die gesamte Lohnentwicklung dient. In einer ersten Auswertung zur Umsetzung des "Global Jobs Pact" hat die ILO insbesondere die Mindestlohnpolitik Brasiliens als positive Anti-Krisenstrategie hervorgehoben (International Institute for Labour Studies/ILO 2009).

In Europa verfolgen derzeit nur wenige Regierungen eine expansivere Mindestlohnpolitik. Dies ist höchst problematisch, weil das relative Mindestlohnniveau in vielen europäischen Ländern immer noch sehr gering ist. Eine strukturelle Erhöhung des Mindestlohnniveaus würde nicht nur vielen Beschäftigten einen angemessenen Lohn oberhalb des Existenzminimums ermöglichen, sondern zugleich auch einen wichtigen Beitrag zur Bekämpfung der Krise leisten. Angesichts des in Europa nach wie vor stark ausgeprägten Lohnkostenwettbewerbs wäre hierzu eine koordinierte Mindestlohninitiative auf europäischer Ebene sicherlich sehr hilfreich (Schulten 2008b). 


\section{LITERATUR}

Australian Council of Trade Unions (ACTU) (2009): Submission to the Australian Fair Pay Commission, Melbourne, März

Australian Fair Pay Commission (2009): Wage-Setting Decision and Reasons for Decision, Juli

Cahuc, P./Cette, G./Zylberberg, A. (2008): Salaire Minimum et Bas Revenus: Comment concilier Justice Sociale et Efficacité Economique? Les Rapports du Conseil d'Analyse Economique 79, La Documentation française, Paris

Conseil d'Orientation pour l'Emploi (COE) (2008): Avis du Conseil sur la Conditionnalité des Allègements de Cotisations Sociales et la Procédure de Fixation du SMIC, 6. Februar

Czech, B. (2009): Minimum Wages in January 2009, Eurostat (Hrsg.): Data in focus 29

da Paz, M./Lima,C./Naumann, R. (2006): Portugal: Social Partners sign Landmark Agreement on Minimum Wage Increase, EIROnline, (www.eurofound.europa.eu/eiro/2006/12/articles/pt0612029i.htm) Dumas, M. (2009): Troisième Année sans Coup de Pouce, Rede auf der Sitzung der Commission Nationale de la Négociation Collective am 23. Dezember (www.cgt.fr/spip.php?page=imprimir articulo\&id_article= 36742)

Eyraud, F./Saget, C. (2005): The Fundamentals of Minimum Wage Fixing Geneva

Filion, K. (2009): A Stealthy Stimulus: How boosting the Minimum Wage is helping to stimulate the Economy, Economic Policy Institute (EPI) Issue Brief 255, 28. Mai

Groupe d'Experts sur le SMIC (2009a): Salaire Minimum Interprofessionnel de Croissance - Rapport Juin

Groupe d'Experts sur le SMIC (2009b): Salaire Minimum Interprofessionnel de Croissance - Rapport Décembre International Institute for Labour Studies (IILS) (2009): World of Work Report 2009. The Global Jobs Crisis and Beyond, Genf International Labour Organization (ILO) (2008): Global Wage Report 2008/09. Minimum Wages and Collective Bargaining: Towards Policy Coherence, Genf
International Labour Organization (ILO) (2009): Recovering from the Crisis: A Global Jobs Pact, adopted by the International Labour Conference at its ninety-eighth Session, Genf, 19. Juni

Jokivuori, P. (2009): Finland: Differing Views of Trade Unions on National Statutory Minimum Wage, EIROnline (www.eurofound.europa.eu/eiro/2009/06/articles/fi0906039i.htm) Kaitz, H. (1970): Experience of the Past: the National Minimum, in: US Department of Labor Bureau of Labor Statistics (Hrsg.): Bulletin 1657, Washington DC, S. 30-54

Krugman, P. (2009): Would cutting the Minimum Wage raise Employment?, in: New York Times Krugman Blog, 16. Dezember (http://krugman. blogs.nytimes.com/2009/12/16/would-cutting-the-minimum-wageraise-employment/)

Low Pay Commission (2009): National Minimum Wage. Low Pay Commission Report 2009

Ministerio de Trabajo e Immigacion (2009): El Gobierno fija el SMI en 633,30 Euros Mensuales, Pressemitteilung vom 30. Dezember Neumark, D. (2009): Delay the Minimum Wage Hike, in: The Wall Street Journal, 12. Juni

Schulten, T. (2008a): Kein Lohn unter 20 Franken! Zum Relaunch der Schweizer Mindestlohnkampagne, in: Sozialismus 6, S. 28-31

Schulten, T. (2008b): Towards a European Minimum Wage Policy? Fair Wages and Social Europe, in: European Journal of Industrial Relations 4, S. 421-439

Schulten, T. (2009): WSI-Mindestlohnbericht 2009, in: WSI-Mitteilungen 3, S. 150-157

Schulten, T. (2010): Das deutsche Tarifvertragsystem im europäischen Vergleich, in: Bispinck, R./Schulten, T. (Hrsg.): Zukunft der Tarifautonomie, Hamburg, S. 193-204

SOLIDAR (2009): Doing a Decent Job? IMF Policies and Decent Work in Times of Crisis (www.eurodad.org/uploadedFiles/Whats_New/Reports/ Doing\%20a\%20decent\%20job.pdf)

Sweeney, P. (2009): Low-paid and Welfare Recipients are the wrong Target, in: Irish Times, 24. Juli 